\title{
Listening Together while Apart: Intergenerational Music Listening
}

\author{
Nabila Chowdhury \\ University of Manitoba \\ Winnipeg, Canada \\ chowdh26@myumanitoba.ca
}

\author{
Celine Latulipe \\ University of Manitoba \\ Winnipeg, Canada \\ celine.latulipe@umanitoba.ca
}

\author{
James E. Young \\ University of Manitoba \\ Winnipeg, Canada \\ young@cs.umanitoba.ca
}

\begin{abstract}
Collaborative music listening can support intergenerational connectedness. We conducted an environmental scan of video conferencing and music listening platforms and cognitive walkthroughs to investigate how these platforms can support collaborative music listening and conversation between a grandparent and teen grandchild. Our results indicate that common video conferencing platforms not only lack a convenient way to share music, but typically block audio from music listening apps played on the same device, preventing sharing. Further, while music streaming platforms enable connected friends to share songs and others' playlists, they do not provide a means for smooth synchronous conversing about the music. Thus, this highlights opportunities for technology improvement to support intergenerational relationships by enabling distributed, intergenerational co-listening of music.
\end{abstract}

\section{CCS CONCEPTS}

- Human-centered computing $\rightarrow$ Collaborative and social computing.

\section{KEYWORDS}

Older Adults; Intergenerational Conversation; Co-listening; Shared Experience; Collaborative Music Listening; Synchronous Communication

\section{ACM Reference Format:}

Nabila Chowdhury, Celine Latulipe, and James E. Young. 2021. Listening Together while Apart: Intergenerational Music Listening. In Companion Publication of the 2021 Conference on Computer Supported Cooperative Work and Social Computing (CSCW'21 Companion), October 23-27, 2021, Virtual Event, USA. ACM, New York, NY, USA, 4 pages. https://doi.org/10.1145/ 3462204.3481765

\section{INTRODUCTION}

Relationships between grandparents and grandchildren offer unique mutual support $[2,6]$, particularly with teenagers $[16,21]$. This interaction is often serendipitous [16], providing a source of stability, mentorship, and encouragement $[6,11]$. Such interactions help older adults view aging more positively [16], provide a means

Permission to make digital or hard copies of all or part of this work for personal or classroom use is granted without fee provided that copies are not made or distributed for profit or commercial advantage and that copies bear this notice and the full citation on the first page. Copyrights for components of this work owned by others than the author(s) must be honored. Abstracting with credit is permitted. To copy otherwise, or republish, to post on servers or to redistribute to lists, requires prior specific permission and/or a fee. Request permissions from permissions@acm.org.

CSCW'21 Companion, October 23-27, 2021, Virtual Event, USA.

(c) 2021 Copyright held by the owner/author(s). Publication rights licensed to ACM. ACM ISBN 978-1-4503-8479-7/21/10 .. \$15.00

https://doi.org/10.1145/3462204.3481765

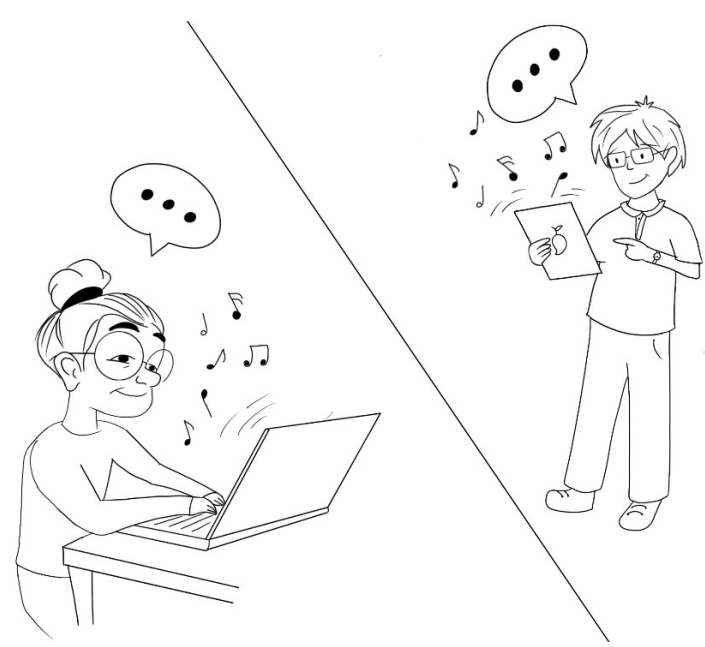

Figure 1: Vision: Grandma and grandson remotely co-listen to music and talk.

to pass on their cultural identity [23], and support general wellbeing [15]. Likewise, maintaining close relationships with grandparents helps to improve mental health in late adolescents and young adults [21].

Maintaining intergenerational relationships remains difficult, for example due to geographical separation [2], lack of common ground [9], scheduling challenges [2, 9], and recently, pandemic related restrictions. We explored the use of technology for supporting such intergenerational relationships. Specifically, we propose synchronous, collaborative music listening as a mechanism for facilitating quality interaction between a grandparent and a teen-aged grandchild (see Fig. 1).

Now-ubiquitous video conferencing platforms (e.g., Zoom, Skype, FaceTime) have emerged as a staple in supporting families separated by geography or pandemic lockdowns. However, as these are commonly designed for work (e.g., Zoom, Skype), or simple video conferencing (e.g., FaceTime), they have limited functionality designed to support more casual intergenerational interaction. Similarly, research has investigated asynchronous platforms to tackle scheduling challenges, such as by enabling one to leave digital messages (e.g., pictures, songs) for remote family members (e.g., [2, 9]). However, asynchronous approaches do not provide the richness and depth of synchronous conversation $[8,17]$.

Collaborative music listening provides an avenue for supporting serendipitous interactions and intergenerational relationships. Music can provide support and create positive emotions (e.g., joy, 
empowerment [1,24]), and is important for both teens [5, 19] and older adults $[3,10,14]$. Sharing music can also help to create feelings of social and emotional connectedness [3, 18], and support interaction within families [13,23], between peers [22], or even between strangers [7].

We propose collaborative music listening over video conferencing as a catalyst for meaningful interaction between a grandparent and teen grandchild. Given the potential benefits to intergenerational interaction, we investigate the feasibility of using technology to support distributed music co-listening over digital platforms. As part of a larger project, we plan to study the interaction challenges relating to generational differences in music taste and content appropriateness, as well as preferred communication patterns and styles, in the context of the intergenerational power and relationship dynamics. But first, we must consider the technical challenges associated with co-listening to music online. In this paper we present results from an environmental scan of existing tools for supporting this, and highlight opportunities for technology to be better designed to support intergenerational, distributed music listening.

\section{ENVIRONMENTAL SCAN OF EXISTING TOOLS}

Jenna and her grandma Lynne are relaxing in Lynne's living room and sharing music. Jenna is playing music from her phone, while Lynne is playing songs on her old record player. They talk over the music, sing along, and share memories sparked by their favorite songs.

This above scenario illustrates fluid in-person interaction; we explored how existing technology can support such interaction online. Prior work explored music-related interventions to support intergenerational communication for physically separated grandparents and grandchildren. Kleinberger et al. [9] facilitated music-triggered conversation between grandparents and adult grandchildren in the Memory Music Box; grandchildren created slides with pictures and music for their grandparents, with a passive grandparent role of only viewing picture slideshows. Tibau et al. [23] explored synchronous music listening to support communication between grandparents and young grandchildren. This project was parentmediated as the parents shared music on behalf of the children, who were between 1.5 to 5 years old. Other research prototypes for co-listening were not explicitly designed to support intergenerational co-listening and so may not translate well without further consideration: PocketSong [7] was designed to enable strangers to casually see what others are listening to, CoListen [22] created a digital space for young people to share music in more isolated semirural areas, and MissU [13] was designed for more intimate sharing of daily experiences within couples. None of these fit our target scenario of focused but distributed co-listening and conversation around music.

Beyond research prototypes, we investigated general purpose popular video-conferencing platforms (i.e., Zoom, Skype, FaceTime) and popular music listening platforms (i.e., Spotify, Apple Music, SoundCloud). We systematically examined these to identify features that can be used to support our target application of geographically separated, intergenerational music co-listening. We installed the selected platforms on a Windows 10 compatible PC and an Apple
iPad, and used paid versions of Spotify and Apple Music to maximize music choice and avoid advertisements. We performed cognitive walkthroughs of music co-listening on these platforms from the perspective of both a grandparent and a teen grandchild.

\subsection{Video Conferencing Platforms}

We explored both desktop and iPad versions of Zoom, Skype and FaceTime. All surveyed platforms supported free-form video conversation. However, we found that the ability to support collaborative music listening hinged on the ability to share audio within the video tool, which none of the surveyed platforms supported.

All three tools (Zoom, Skype, and FaceTime) enable people to share music external to the device, for example, if played from a home stereo or different computer, by transmitting music along with the people's voices. The only tool that supported sharing of music from the same device was Zoom (5.6.1, Windows 10, 2021). The desktop version of Zoom allows users to share their screen along with audio, so that when playing a video or audio file in any application, the remote person can hear it. However, this requires users to know this can be enabled, and how to do it. Zoom can share the device audio only (without sharing the screen), which requires the sharing user to use advanced settings. The situation is similar on the iPad (5.6.6, 8th generation, 2021), except it does not support the audio-only sharing option.

Skype (8.72.0.94, Windows 10, 2021) does not have an audiosharing feature. By default, if one plays audio from the same machine (e.g., in a music player) Skype's noise cancellation technology filters the audio out. Skype suggests setting the noise cancellation level to 'low' to share music, but the audio is poor and cuts out. On an iPad, Skype has no noise cancellation setting and so device audio is always filtered out (8.72.0.96, iPad 8th generation, 2021).

Currently, FaceTime (iOS/iPadOS 14.6, iPad 8th generation, 2021) does not support screen or audio sharing. Similar to Skype, if a user plays audio on the same device while on FaceTime, the device filters it out and does not send it to the remote person. Further, on the iPad version, FaceTime video stops while the user is in other applications.

\subsection{Music Platforms}

Music platforms, by design, enable a person to play a desired song; in this section we explore what features they have that can support our distributed co-listening scenario. All platforms we explored - Spotify (8.6.30.968, iPad 8th generation, 2021), Apple Music (iOS/iPadOS 14.6, iPad 8th generation, 2021), and SoundCloud (77060 iPad 8th generation, 2021) - have built in support for sharing music links. These features enable users to link these accounts to social media platforms such as Facebook, and to add other people in the same system as friends. Being a 'Friend' on Spotify, Apple Music, or SoundCloud allows users to see what their friends and family members are listening to and supports creating collaborative playlists. This requires both users to have accounts on the same platform and to be linked as friends. Spotify recently added a 'Group Session' feature in their premium subscription (mobile only), that enables a group of 2-5 distributed people to synchronously listen. Everyone in the group can pause, play, skip and select tracks from the playlist and such changes are immediately reflected on all 


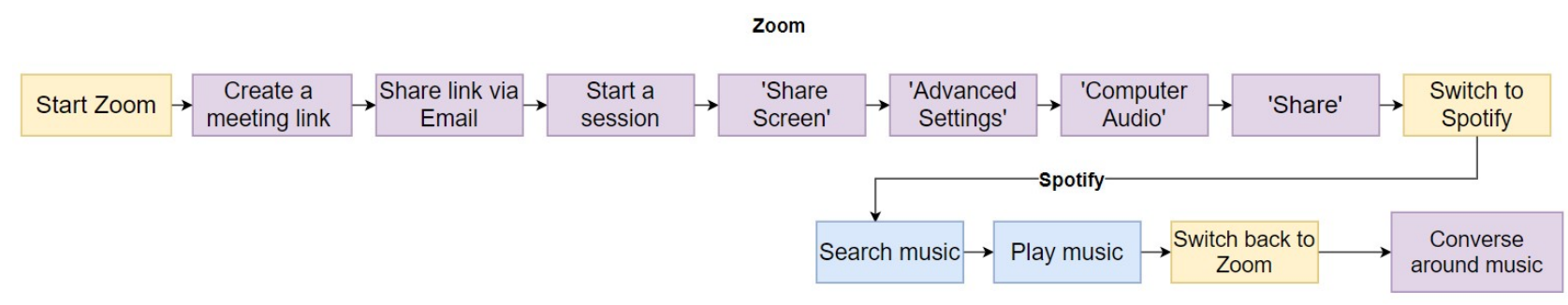

Figure 2: Steps needed to co-listen and converse around music using Zoom and Spotify.

participant devices. However, participants have to share the group link using messaging apps or social media. Apple Music and SoundCloud do not support any such features at this time. None of these platforms support synchronous communication between users via text, video or audio chat, so there is no way to have conversations with the music playing, without using some other communication channel.

All three platforms support sharing by providing links to specific songs. Links can be a useful way to share songs, but require use of an external mechanism for link sharing (e.g., email, SMS, or a social form). Alternatively, users always can verbally share a song name or artist for the other person to search on their own system. All of these approaches take time and introduce potential pinch points in communication, which may hinder co-listening.

As above, playing music on a device while video conferencing is not trivial, as the music is often filtered from the transmitted audio. Further, not everyone is connected via social networking, and not all systems support chat (e.g., FaceTime) to send links or song names; if they do, copy-pasting a link between applications can be challenging.

\subsection{Sample Walkthroughs}

Our approach is inspired by cognitive walkthroughs, where we focus on what the actual users may want to do, what they understand, etc. $[12,20]$, to understand what it takes for a grandparent and a teenage grandchild to use these technologies to co-listen to music and converse around it, and what barriers they may face in doing so. We conducted our walkthroughs with various technology configurations, with the task of having an online conversation between two users, with each sharing a favorite song. We share summaries of two of these walkthroughs here.

Our first technology configuration was Zoom and Spotify on a desktop and an iPad. First, User1 created a meeting link and shared it with User2 via email. After User2 joined Zoom, User1 decided to share audio from their desktop computer. To do so, User1 clicked on the 'Share Screen' button and then clicked on the 'Computer Audio' button and then the 'Share' button. After that, User1 opened Spotify. In Spotify, User1 searched for their song and then clicked the 'Play' icon. While the music was playing, User1 and User2 conversed about the song. After the song, User2 tried to play a piece of music from their iPad via Zoom. When User2 tried to share the screen, they found there was no option in Zoom on the iPad to share only audio from the device. So, User2 shared the iPad screen and then played a song. On the iPad, Zoom does not allow sharing only a portion of the screen, so while User2 was looking for a song on
Spotify, User1 was able to see all the detail of User2's playlists on the screen. After listening to the music, the users talked and then ended the Zoom session. Fig. 2 provides a visualization of these steps.

Our second technology configuration used FaceTime and Apple Music on iPad. User1 launched a FaceTime session with User2. After greetings, User 1 tried to play a song via Apple Music on iPad. When User1 was out of FaceTime to open Apple Music, their video was paused for User2. The iPad did not offer a minimization option for FaceTime, but User2 could still hear User1. After User1 found the song they wanted to share, they clicked the 'Play' icon on Apple Music. However, User2 did not hear any music. Then User1 decided to play the music from their iPhone using Apple Music (by keeping the iPhone very close to the first device and putting the music on the loudspeaker). While this worked, the sound quality was very poor for User2. User2 also tried to share music via Apple Music and the same situation occurred. In the end, each user created a playlist and shared it with the other via iMessage (as FaceTime does not support text chat) on their iPad. The users then ended their session and listened to the music separately.

While we want to support music as a catalyst to the conversation between older adults and teenage grandchildren, none of these platforms supported such shared experiences.

\section{BARRIERS \& OPPORTUNITIES FOR INTERGENERATIONAL CO-LISTENING}

If we consider a grandparent and grandchild sitting together in a living room and listening to music while talking, none of the technology configurations examined came close to supporting such fluid face-to-face interaction. We have broadly categorized the potential barriers that can disrupt the conversation flow as technical versus contextual issues.

\subsection{Technical Issues around Music Sharing}

To co-listen to music on Zoom, FaceTime or Skype, family members either have to share music links on the platform or play a song on a loudspeaker so that the other user can listen to the music. Searching for a song link in the middle of the conversation can be time-consuming for some users due to searching on multiple platforms (e.g. Spotify, YouTube Music, Apple Music) or not finding the correct songs (e.g. same songs but different covers, and mixes). Pausing the conversation and looking for songs can hinder the conversation flow between grandparents and teen grandchildren. This is especially true if the person searching is not sharing their screen, 
as what they are doing is unseen by the other user, and the other user can not help and may feel left out. Similarly, if music is shared on a loudspeaker, there is a possibility the video platforms will identify such music as environmental noise and may filter out the songs. Only desktop Zoom allows music from another application to be directly piped in, but requires advanced settings manipulation. The music streaming services do not typically support co-listening and do not support conversation between users.

\subsection{Contextual Issues to Spark Conversation}

Current online communication platforms do not have designed features for proactively encouraging the kind of emotional engagement and playful interaction so important for grandparents and grandchildren. We have identified music as one such catalyst, and suggest that technologies connecting grandparents and teen grandchildren could offer more support for quality interaction (e.g., as in [4]). To support sustained, rich interaction between grandparents and teen grandchildren, we suggest that new video-conferencing technology designs in this space could offer the following:

- simple mechanisms to easily share media and co-watch or co-listen, while still video-conferencing

- interaction prompts that allow discussion/highlighting of different parts of the media, to deepen conversation

- prompts for memory sharing and recording, or ways to cojournal

\section{CONCLUSION AND FUTURE WORK}

We conducted an environmental scan of existing video conferencing tools and music streaming platforms in the context of supporting intergenerational co-listening and discussion of music. We found most of the common video conferencing platforms do not support a convenient way to play music. The steps a grandparent and grandchild dyad has to follow to share computer audio for co-listening can hinder the enthusiasm to listen to music together. Currently, available music streaming platforms also do not support a direct way to have a conversation (synchronous or asynchronous). However, platforms such as Spotify are beginning to incorporate more social components (e.g., group sessions to co-listen to music) in their platform.

Our goal is to support the serendipitous interaction that could occur face-to-face while sharing music between grandparents and teen grandchildren, just like the scenario where Jenna and her grandma Lynne share music and talk comfortably with one another. While asynchronous communication can help people stay in touch, we are more interested in finding how synchronous communication can offer a rich shared intergenerational experience, specifically for grandparents and teen grandchildren. With our future work, we hope to contribute a deep understanding of how older adults and teen grandchildren converse around music and consider how we can use technology to facilitate their interaction around music. This will help us to gain insights into the design goals for technology that can support and sustain rich connectedness between grandparents and their teen grandchildren.

\section{REFERENCES}

[1] Susan Bluck and Linda J Levine. 1998. Reminiscence as autobiographical memory: A catalyst for reminiscence theory development. Ageing and Society 18, 2 (1998), 185-208.

[2] Mark Butzer, Zachary Levonian, Yangyang Luo, Kathleen Watson, Ye Yuan, C Estelle Smith, and Svetlana Yarosh. 2020. Grandtotem: Supporting International and Intergenerational Relationships. In Conference Companion Publication of the 2020 on Computer Supported Cooperative Work and Social Computing. 227-231.

[3] Ayelet Dassa and Dorit Amir. 2014. The role of singing familiar songs in encouraging conversation among people with middle to late stage Alzheimer's disease. Journal of music therapy 51, 2 (2014), 131-153.

[4] Bente Evjemo, Gunnvald B Svendsen, Eivind Rinde, and Jan-Are K Johnsen. 2004. Supporting the distributed family: the need for a conversational context. In Proceedings of the third Nordic conference on Human-computer interaction. 309-312.

[5] E Fiossi-Kpadonou, DV Sessou, GT Kpadonou, and T Agossou. 2016. Music and emotions of teenagers in Benin. Fournal of Child and Adolescent Behavior (2016), $1-7$.

[6] Candace L Kemp. 2005. Dimensions of Grandparent-Adult Grandchild Relationships: From Family Ties to Intergenerational Friendships. Canadian fournal on Aging/La Revue canadienne du vieillissement 24, 2 (2005), 161-177.

[7] David S Kirk, Abigail Durrant, Gavin Wood, Tuck Wah Leong, and Peter Wright. 2016. Understanding the sociality of experience in mobile music listening with Pocketsong. In Proceedings of the 2016 ACM Conference on Designing Interactive Systems. 50-61.

[8] David S Kirk, Abigail Sellen, and Xiang Cao. 2010. Home video communication: mediating'closeness'. In Proceedings of the 2010 ACM conference on Computer supported cooperative work. 135-144.

[9] Rebecca Kleinberger, Alexandra Rieger, Janelle Sands, and Janet Baker. 2019. Supporting Elder Connectedness through Cognitively Sustainable Design Interactions with the Memory Music Box. In Proceedings of the 32nd Annual ACM Symposium on User Interface Software and Technology. 355-369.

[10] R Kneafsey. 2005. Therapeutic Music Research Abstracts-Gerontology. Psychology of Music 33, 4 (2005), 437-451.

[11] Arthur Kornhaber and Kenneth L Woodward. 1981. Grandparents, grandchildren: The vital connection. Transaction Publishers.

[12] Clayton Lewis, Peter G Polson, Cathleen Wharton, and John Rieman. 1990. Testing a walkthrough methodology for theory-based design of walk-up-and-use interfaces. In Proceedings of the SIGCHI conference on Human factors in computing systems. 235-242.

[13] Danielle Lottridge, Nicolas Masson, and Wendy Mackay. 2009. Sharing empty moments: design for remote couples. In Proceedings of the SIGCHI conference on Human factors in computing systems. 2329-2338.

[14] Robin A Majeski and Merrily Stover. 2019. The expressive arts and resilience in aging. Educational Gerontology 45, 3 (2019), 161-166.

[15] Lois J McCloskey. 1985. Music and the frail elderly. Activities, Adaptation \& Aging 7, 2 (1985), 73-75.

[16] Karyn Moffatt, Jessica David, and Ronald M Baecker. 2013. Connecting grandparents and grandchildren. In Connecting Families. Springer, 173-193.

[17] Carman Neustaedter, Carolyn Pang, Azadeh Forghani, Erick Oduor, Serena Hillman, Tejinder K Judge, Michael Massimi, and Saul Greenberg. 2015. Sharing domestic life through long-term video connections. ACM Transactions on ComputerHuman Interaction (TOCHI) 22, 1 (2015), 1-29.

[18] Kenton O'Hara and Barry Brown. 2006. Consuming music together: social and collaborative aspects of music consumption technologies. Vol. 35. Springer Science $\&$ Business Media.

[19] Zoe E Papinczak, Genevieve A Dingle, Stoyan R Stoyanov, Leanne Hides, and Oksana Zelenko. 2015. Young people's uses of music for well-being. fournal of Youth Studies 18, 9 (2015), 1119-1134.

[20] Peter G Polson and Clayton H Lewis. 1990. Theory-based design for easily learned interfaces. Human-Computer Interaction 5, 2-3 (1990), 191-220.

[21] Sarah A Ruiz and Merril Silverstein. 2007. Relationships with grandparents and the emotional well-being of late adolescent and young adult grandchildren. Journal of Social Issues 63, 4 (2007), 793-808.

[22] Michael Stewart, Javier Tibau, Deborah Tatar, and Steve Harrison. 2018. Codesigning for co-listening: Conceptualizing young people's social and musiclistening practices. In International Conference on Social Computing and Social Media. Springer, $355-374$.

[23] Javier Tibau, Michael Stewart, Steve Harrison, and Deborah Tatar. 2019. FamilySong: Designing to enable music for connection and culture in internationally distributed families. In Proceedings of the 2019 on Designing Interactive Systems Conference. $785-798$.

[24] Melissa K Weinberg and Dawn Joseph. 2017. If you're happy and you know it: Music engagement and subjective wellbeing. Psychology of Music 45, 2 (2017), 257-267. 